\title{
ELECTROMAGNETIC MODELLING FOR INFORMATION EXTRACTION FROM HIGH RESOLUTION SAR IMAGES OF URBAN AREAS
}

\author{
Giorgio Franceschetti, Raffaella Guida, Antonio Iodice, Daniele Riccio
Dipartimento di Ingegneria Elettronica e delle Telecomunicazioni
Università di Napoli Federico II
Via Claudio 21 - 80125 Napoli
\{gfrance, rafguida, iodice, daniele.riccio\}@unina.it

\begin{abstract}
Analysis, interpretation and feature extraction concerning High Resolution (HR) Synthetic Aperture Radar (SAR) images of urban areas urgently require support of sound and appropriate electromagnetic modelling.

The modelling takes into consideration the radar geometry and the (geometric and electromagnetic) scene parameters but also the novelty brought by high resolution.

In this paper, this way of developing suitable electromagnetic modelling for HR SAR images of urban areas is shown to be successful as able to interpret and retrieve, from these scenarios, new and interesting details that will certainly represent the main actor of next generation of applications for urban areas with SAR sensors.
\end{abstract}

Index Terms - Synthetic Aperture Radar (SAR), high resolution, urban areas, modelling, feature extraction.

\section{INTRODUCTION}

The launch of new satellites hosting High Resolution (HR) Synthetic Aperture Radar (SAR) sensors has certainly been one of the most interesting novelties in the last year in the field of microwave remote sensing 0 .

SAR images with 1 meter resolution are completely changing our approach of interpretation of any kind of scene. Whether we consider a natural scenario or a built-up area, high resolution deeply affects the relative SAR image and often confuses and mystifies comprehension for user accustomed to standard spaceborne SAR products with resolution of tens of meters. As a matter of fact, users of standard resolution SAR products might think that HR products allow a "better" view of the scene, whereas HR resolution images allow to view "more" details of the observed scene.

These considerations are particularly true and become transparent for urban areas where the radar return of a resolution cell can be dominated by man-made objects whose dimensions are significantly smaller than the resolution cell; this is not the case for natural landscapes.
For this reason, urban areas need sound and tailored electromagnetic models able to well represent the interactions between the radar signal and the urban structures. However, model-based approaches including a reliable electromagnetic modelling are rarely presented in the literature: simple geometric models are in general introduced. These models do not fully explain the SAR image features as far as also layover and shadowing effects change their appearance in HR SAR images of urban areas or man-made objects. Moreover, surfaces roughness and buildings materials are poorly or not considered at all, thus neglecting the important role they play in the SAR image formation.

In conclusion, it is then mandatory to provide an electromagnetic model so that even for simplified structures, any feature on HR SAR images can be explained [2, 3].

In this paper we introduce original electromagnetic models to investigate how different resolutions can affect the formation of the SAR images.

These models are based on previous modelling developed for canonical structures [2] but now the structures are complicated to take into account fine details like windows and balconies. In fact, as will be shown in the following, high resolution allows to detect these parts in a building in many cases and, consequently, their consideration in the phase of modelling is becoming mandatory for the important applications that can be derived.

The proposed models apply under a wide range of hypothesis, thus they are not simply based on the phenomenological observation of SAR images, the latter being an approach that could lead to considerations that are only true for the case at issue. The proposed models rely, instead, on the quantitative description of the scattering mechanisms produced by the most common geometries appearing in urban areas. With this approach, appropriate and efficient expressions describing the electromagnetic behavior of plane surfaces, dihedral and trihedral configurations may be derived, employed and applied to the urban area constituents: here, in particular, the ratio between the backscattering coefficients relevant to the contributions coming from these structures are written in terms of the of 
the SAR range and azimuth resolutions. In this way, for the same structure, any change happening in a SAR image with the increasing resolution can be evaluated.

After that, at different levels of resolution the appearance of a simple geometric structure in a SAR image is quantitatively forecast: each expected contribution is not only located in the relevant range position, but also numerically evaluated in extension along range and azimuth and grey-level intensity.

The effort is meaningful if we care to find efficient and general tools for the interpretation of this kind of image. A practical example is also analyzed thus showing the guidelines for a correct and full interpretation of HR SAR images of simple urban structures.

\section{MODELS FOR HR SAR IMAGES}

High Resolution SAR images show buildings in a very detailed representation which needs models to be analyzed. Models for urban areas are an essential support, above all if we consider that these areas are those which more benefit of high resolution. But the interpretation of HR SAR images is not trivial for many reasons and, among the others, because we can not rely our analyses on previous existing models without bringing some important improvements to them. This need arises from the following considerations.

In built-up areas geometries like flat planes, dihedrals and trihedrals are overabundant: it should be sufficient to think, for example, of roofs, walls and the geometries they form with the ground or close surfaces. Now, in literature references can be found about the scattering of this kind of structures $[4,5]$ and its dependence on working frequency or the structure dimensions. As a first consideration, simply having a look to those formulas we can then derive that, thanks to the better resolution, in HR SAR images dihedrals and trihedrals should appear generally brighter than flat plans. In fact, if we consider for example a building with a wall parallel to the flight trajectory, we know that the backscatter cross section $\sigma_{d}$ of the dihedral formed by the wall and the ground, evaluated in the high frequency range with geometric optics, is given by [4,5]

$\sigma_{d} \propto 4 \pi A_{e}^{2} / \lambda^{2}$

where $A_{e}$ is the effective aperture of the corner reflector, obviously related to the dimensions of the surfaces involved and the radar look angle, and $\lambda$ is the working wavelength. Now, the backscattering of wall and roof is backscattering from flat planes which only in the case of normal incidence is

$\sigma_{p n}=4 \pi A^{2} / \lambda^{2}$

where now $A$ is the real area of the plane. But in most cases, instead, the incidence is not normal, like the example at hand. In this case, in which one of the plane sides is parallel to the line of flight, the backscattering cross section is

$\sigma_{p} \propto 4 \pi b^{2}$

where $b$ is the length of the parallel side.

Now, with improving resolution, each length is better represented and, consequently, areas are those which benefit more. In turn, all backscattering depending upon two dimensions, instead of only one, profit in a more significant way from higher resolutions. If we consider the ratio between (1) and (3) and suppose that one of $A_{e}$ dimensions is $b$,

$$
\frac{\sigma_{d}}{\sigma_{p}} \propto A_{e}^{2} / b^{2}=\frac{(a * b)^{2}}{(b)^{2}}=a^{2}
$$

that should be the theoretical relation between those scattering contributions. Actually, on the SAR image, we have a different fraction as the dimensions involved are only partially represented, because of resolution, and above ratio should be:

$$
\frac{\sigma_{d}}{\sigma_{p}} \propto \frac{\left(n_{b} \Delta x * n_{a} \Delta r\right)^{2}}{\left(n_{b} \Delta x\right)^{2}}=n_{a}^{2} \Delta r^{2}<a^{2}
$$

where $\Delta x$ and $\Delta r$ are, respectively, the SAR image resolution in azimuth and slant range, and $n_{b}$ and $n_{a}$ are the numbers of resolution cells contained respectively in $b$ and $a$.

The better the resolution, the closer is the scattering ratio to its theoretical value in eq.(4).

Trihedral geometries present a backscattering that also depends on the fourth power of a length (that of one edge of the trihedral aperture whatever is the type: square, triangular or circular, [4]) and then similar considerations can be derived.

A second consideration is mainly linked to a geometrical matter: with the resolution improvement every contribution is better separated from the surroundings and this has also an important consequence on the SAR image appearance above all when the interpretation is based on models. In fact, in this case each element in the scene produces its own contribution (result of the geometric and electromagnetic properties of the element) which is not melt with the others and, being distinguishable, can be extracted and considered for the image analysis.

In the next section a HR SAR image of urban areas is analyzed and discussed with emphasis to novelties introduced by a high resolution.

\section{ANALYSIS AND MEASURE OF HR SAR IMAGES}

SAR images with geometric resolution in the order of 1 meter are finally available. Fig. 1 shows a particular of the High Spotlight TerraSAR-X image acquired on 12 May 


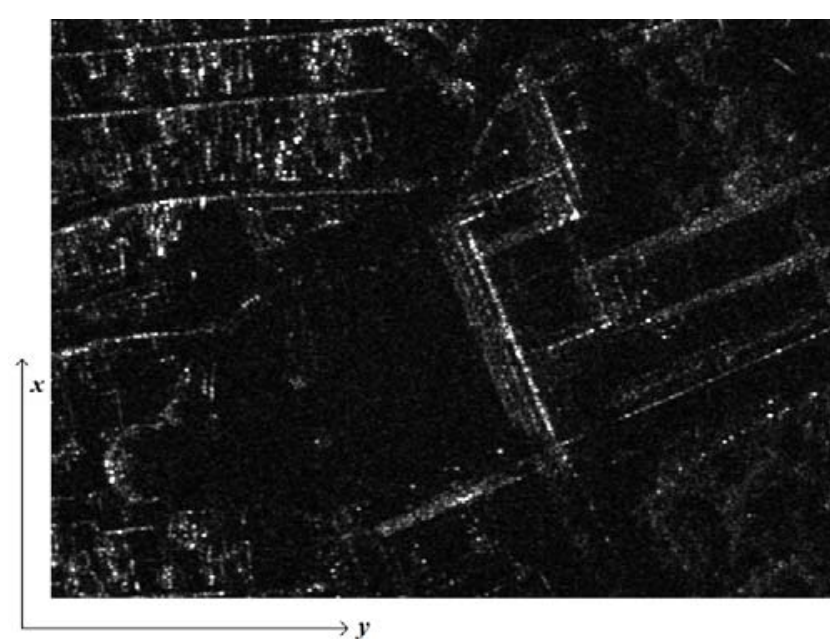

Figure 1 - High Spotlight TerraSAR-X image of Piazza del Plebiscito, Naples. (C) DLR (2008).

2008 on the city of Naples: Piazza del Plebiscito is clearly represented with Palazzo Reale on the right side. SAR image resolutions in azimuth and ground range are: $\Delta x=1.255 \mathrm{~m}$ and $\Delta y=1.486 \mathrm{~m}$. An aerial view of the square is also provided in Fig. 2 while, in Fig.3, the main façade of Palazzo Reale is shown.

The visit of the site allowed to collect information about the building façade which clearly shows the presence of two long balconies and a gabled roof. From optical images the height of each floor has been retrieved and corresponds to $10 \mathrm{~m}, 9 \mathrm{~m}$ and $7.5 \mathrm{~m}$ respectively for the ground, the first and the second floor.

It is interested to note that in the SAR image more than one bright line is present in the appearance of the main façade of Palazzo Reale. Should not be clear at a first glance, it is certainly after a radiometric analysis of the SAR image: a plot profile is given in Fig.4 where four peaks are distinguished. The higher peak, corresponding to the brightest line, is also the most far in range and has been related to the double reflection contribution.

In low resolution SAR images, we would have found a very different profile with only one dominant larger peak preceded by a more homogeneous area known as layover. Thanks to the high resolution, instead, a new information content is now provided.

The other three peaks may be related, in order from right to left in Fig.4, respectively to the banister of the long balcony at the first floor (and not to the dihedral configuration presented there as, according to the electromagnetic model in [2], the relative contribution should be much smaller being directly proportional to the height of corner reflector), to the same effect at the second floor and to the edge formed by the façade and the roof. To prove this hypothesis, measures have been taken on the SAR image and then compared with the expected ones deriving from the models

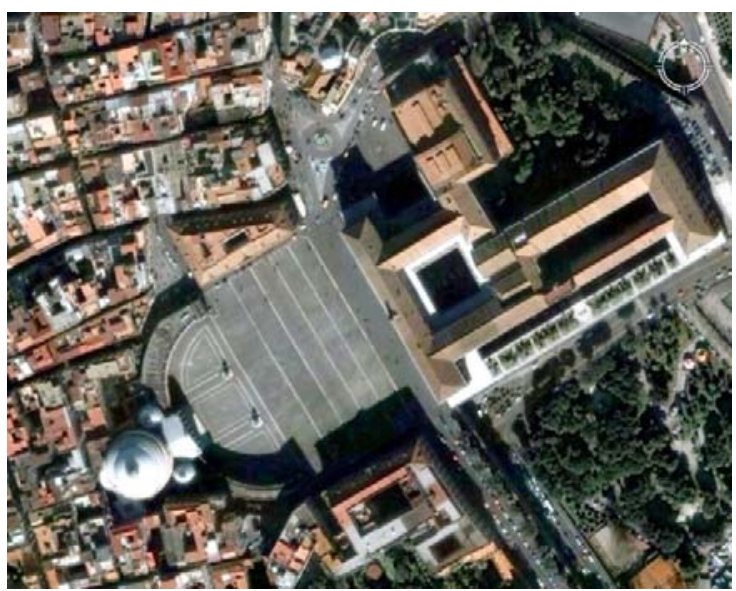

Figure 2 - Aerial view of Piazza del Plebiscito, Naples. (C) 2008 DigitalGlobe.

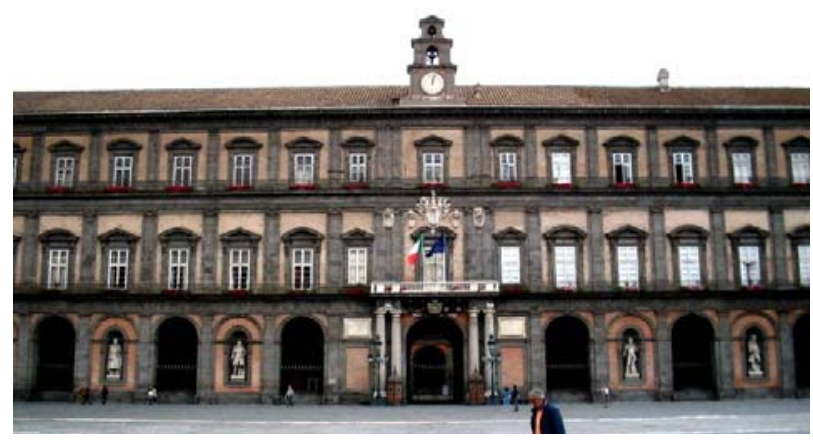

Figure 3 - Main facade of Palazzo Reale, Napoli.

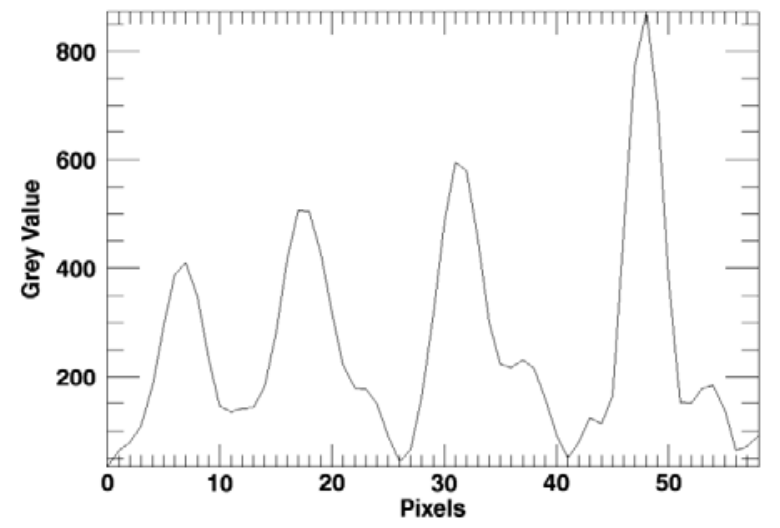

Figure 4 - Plot profile of the main facade of Palazzo Reale, Napoli.

adapted to the building geometry and the radar parameters. In fact, in the above hypothesis, the distance $d$ between the peaks (or two bright lines) is directly linked to the height of each floor $h_{f}$ being:

$h_{f}=d * \tan \vartheta$ 
where $\vartheta$ is the radar look angle (53.5 $5^{\circ}$ in the case at issue). The approach is interesting as it suggests that numbering the floors of a building and retrieving the relative height is possible.

To evaluate the relative distance between two peaks, a narrow and long Region of Interest (ROI) has been cut close to each bright line and moved around until it reached the maximum mean value of grey level in the statistical analysis. When this happened, the bright line was considered localized. The relative distance in pixel was converted in meters being known the pixel spacing of the SAR image $(0.5 \mathrm{~m} \times 0.5 \mathrm{~m})$ and then, using eq.(6) in the floor height.

In this way we retrieved the heights $\hat{h}_{f}$ in Table I where the real floor heights are also reported together with the error $e=h_{f}-\hat{h}_{f}$. We note that each error is always smaller than the resolution in the vertical plane, i.e. $\Delta z=\Delta y^{*} \tan \vartheta$ $=2.008 \mathrm{~m}$ with which the retrieved heights should be compared. In particular, the best height retrieved is that relevant to the ground floor (as its ground range projection is, at least on one side, clearly delimited by the very bright double reflection line), while the errors on the first and second floor height quite compensate each other (the localization of the bright line "shared" by their ground range projections is probably worsen because the backscattering of the gabled roof also falls in that region).

The results are interesting and do not represent an isolated case. On many other sites the authors are working to show the potentiality of high resolution SAR images on urban areas especially when electromagnetic models developed $a d$ hoc are employed to extract features from SAR images. Further examples will be discussed at the conference.

\section{CONCLUSIONS}

In this paper, a feature extraction approach based on models has been shown to fit the complex, but rich in information content, reality of high resolution SAR images of urban areas.

The electromagnetic models, developed for canonical structures, proved to be able to include, and then explain, also effects deriving by the novelty of better resolution of the order of 1 meter: in a building the presence of balconies is detected, the number of floors is determined as well as the height of each of them.

This is possible thanks to the changes brought by the high resolution to the SAR image appearance which the models are able to intercept: each scattering is better radiometrically represented and geometrically confined.

A practical example has been discussed in details confirming not only the effectiveness of the models but also the huge and appealing applications that can be derived.
TABLE I

\begin{tabular}{|c|c|c|c|}
\hline & $h_{f}$ & $\hat{h}_{f}$ & $e=h_{f}-\hat{h}_{f}$ \\
\hline Ground floor & $10 \mathrm{~m}$ & $9.95 \mathrm{~m}$ & $0.05 \mathrm{~m}$ \\
\hline First floor & $9 \mathrm{~m}$ & $9.95 \mathrm{~m}$ & $-0.95 \mathrm{~m}$ \\
\hline Second floor & $7.5 \mathrm{~m}$ & $5.97 \mathrm{~m}$ & $1.53 \mathrm{~m}$ \\
\hline
\end{tabular}

\section{ACKNOWLEDGEMENT}

The research presented in this paper has been carried on within the project S.Co.P.E of University Federico II of Naples and thanks to the TerraSAR-X images provided by the German Aerospace Center (DLR) for the activity of proposal ID MTH0183 in the TerraSAR-X Announcement of Opportunity.

\section{REFERENCES}

[1] R.Werninghaus, S.Buckreuss, W.Pitz, "TerraSAR-X Mission Status", Proceedings of the International Geoscience and Remote Sensing Symposium, Barcelona, pp.3927-3930,2007.

[2] G.Franceschetti, A.Iodice, D.Riccio, “A Canonical Problem in Electromagnetic Backscattering from Buildings”, IEEE Trans. Geosc. Remote Sensing, vol.40, pp.1787-1801, 2002.

[3] R.Guida, A.Iodice, D.Riccio, U.Stilla, "Model-Based Interpretation Of High Resolution SAR Images Of Buildings", IEEE Journal of Selected Topics in Applied Earth Observations and Remote Sensing, in print.

[4] G.T.Ruck, D.E.Barrick, W.D.Stuart, C.K.Krichbaum, Radar Cross Section Handbook, Volume 2, Plenum Press, New York, 1970.

[5] E.F.Knott, J.F.Shaeffer, M.T.Tuley, Radar Cross Section, $2^{\text {nd }}$ Edition, SciTech Publishing, NC, 2004. 